Rev. Int. Contam. Ambie. 37, 249-257, 2021

https://doi.org/10.20937/RICA.53643

\title{
SISTEMA ACOPLADO REACTOR ANAEROBIO CON DEFLECTORES-HUMEDAL ARTIFICIAL COMO ALTERNATIVA PARA REÚSO DE AGUA RESIDUAL EN RIEGO DE ÁREAS VERDES
}

Coupled UASB-constructed wetland system for reuse of wastewater for gardening irrigation

\author{
David HERRERA-LÓPEZ ${ }^{1 *}$, Gamaliel MEJÍA-GONZÁLEZ ${ }^{1}$, Raúl CUEVAS-GONZÁLEZ1 ${ }^{\text {, }}$ \\ Mauricio Antonio ARÉVALO-VELÁZQUEZ ${ }^{2}$ y Griselda Karina GUILLÉN-NAVARRO ${ }^{1}$
}

${ }^{1}$ El Colegio de la Frontera Sur Unidad Tapachula, km 2.5 Carretera Antiguo Aeropuerto, 30700 Tapachula, Chiapas, México.

${ }^{2}$ Instituto Tecnológico de Tapachula, km. 2 Carretera a Puerto Madero, Centro, 30700 Tapachula, Chiapas, México.

*Autor para correspondencia: dherrera@ecosur.mx

(Recibido: junio de 2019; aceptado: julio de 2020)

Palabras clave: reactor con deflectores, reúso de agua, tratamiento terciario

\section{RESUMEN}

Se utilizó un sistema acoplado reactor anaerobio con deflectores-humedal artificial para el tratamiento de agua residual proveniente de un centro de investigación, que por su origen tiene características diferentes al agua residual doméstica. El reactor anaerobio se utilizó a escala completa $\left(20 \mathrm{~m}^{3}\right)$ y el humedal artificial con plantas de caña de azúcar y heliconias a escala piloto $\left(1.36 \mathrm{~m}^{3}\right)$. La eficiencia se determinó en términos de la remoción de materia orgánica medida como demanda química de oxígeno (DQO) y demanda bioquímica de oxígeno $\left(\mathrm{DBO}_{5}\right)$, sólidos suspendidos (SST) y organismos coliformes totales y fecales. A los 140 días de operación se logró la menor concentración de materia orgánica medida como $\mathrm{DBO}_{5}$ en el efluente del sistema, que fue de $22 \mathrm{mg} / \mathrm{L}$. Ésta fue ligeramente superior a la norma mexicana para riego de áreas verdes con contacto directo, aunque cumple con la norma europea Clase B, es decir, para riego de cultivos alimentarios que no entren en contacto con el agua, para riego de alimentos que serán procesados o para riego de alimentos para ganado. La remoción de SST y organismos coliformes totales alcanzada con el sistema fue superiores al $90 \%$, por lo cual se compara favorablemente con sistemas similares. Las concentraciones de salida lograron cumplir normas nacionales y la norma europea. Con el sistema acoplado reactor anaerobio con deflectores-humedal artificial fue posible remover SST y organismos coliformes fecales cumpliendo con las normas europea y mexicana vigentes para el riego en áreas verdes; sin embargo, requiere mejoras en cuanto a la salida de materia orgánica medida como $\mathrm{DBO}_{5}$.

Key words: baffled reactor, reuse of wastewater, tertiary treatment

\begin{abstract}
A coupled anaerobic baffled reactor-constructed wetland system was used for the treatment of wastewater from a research center, whose composition is different from
\end{abstract}


domestic wastewater due to its origin. The anaerobic baffled reactor was used at full scale $\left(20 \mathrm{~m}^{3}\right)$ while the constructed wetland with sugar cane and heliconia plants was used at pilot scale $\left(1.36 \mathrm{~m}^{3}\right)$. System efficiency was measured in terms of organic matter removal as chemical oxygen demand (COD) and biochemical oxygen demand $\left(\mathrm{BOD}_{5}\right)$, total suspended solids (TSS) and total and fecal coliforms. The lowest organic matter concentration of $22 \mathrm{mg} / \mathrm{L}$ was found at day 140 in the system effluent; it was slightly higher than the Mexican regulation for gardening irrigation, although it complies with the European regulation for water B class that can be used for irrigation of alimentary crops which are not in contact with the water, irrigation for crops for processed foods or irrigation for cattle food. The removal of TSS and coliforms with the system was higher than $90 \%$, thus it compares well with similar systems and effluent concentrations and complies with European and Mexican regulation for gardening irrigation. Concentrations of organic matter such as $\mathrm{COD}$ and $\mathrm{BOD}_{5}$ in the effluent of the wetland were $22 \mathrm{mg} / \mathrm{L}$ (slightly higher than Mexican regulation but compliant with the European regulation for water class B) at 140 days of operation. Concentrations of TSS $(10 \mathrm{mg} / \mathrm{L}$ ) and total coliforms (93 MPN/100 mL), positively compared with other systems and complied with the European and Mexican regulations. Using the coupled anaerobic baffled reactor-constructed wetland system it was possible to remove TSS and coliforms in compliance with European and Mexican regulations for gardening irrigation; however, organic matter removal as $\mathrm{BOD}_{5}$ must be improved.

\section{INTRODUCCIÓN}

Según reportes de la Organización de las Naciones Unidas (ONU), alrededor de 500 millones de personas viven en sitios donde el consumo de agua excede la oferta (WWAP 2017), lo que genera un desequilibrio o escasez del líquido en esos lugares. Debido a lo anterior, diversos países han comenzado a implementar el uso de tecnologías de tratamiento de agua residual con la meta de reutilizar el agua tratada y resolver el problema de escasez de agua que actualmente enfrentan (Maryam y Büyükgüngör 2019).

Se han implementado diferentes tipos de tratamientos terciaros, como métodos abióticos (Hamoda et al. 2004) y biológicos (Dong-Qing et al. 2015) en agua residual municipal, los cuales permiten obtener niveles adecuados de materia orgánica para el reúso de aguas residuales. Por las características propias de la generación de agua residual, se debe buscar la tecnología adecuada para cada caso particular. En el caso de un centro de investigación, el agua residual es una mezcla compleja, distinta a las aguas domésticas debido a que, en este tipo de instituciones, los efluentes provienen de sanitarios, laboratorios, comedores o cafeterías y, de manera ocasional, habitaciones. Aunque en laboratorios se llevan a cabo protocolos para la eliminación adecuada de residuos químicos, el lavado de materiales y otras actividades, estos sitios pueden aportar al agua una mezcla variada de sustancias, entre ellas residuos de reactivos que pueden complicar su tratamiento. Por su complejidad y por la calidad exigida para el reúso del agua, de manera general ésta requiere de algún tratamiento terciario que puede incluir entre otros, desinfección (Salgot y Folch 2018). En México el agua que se reúsa en riego de áreas verde debe cumplir con los parámetros establecidos en la NOM-003-SEMARNAP-1997 (SEMARNAP 1998), la cual establece los límites máximos permisibles de contaminantes para aguas residuales tratadas que se reúsan en el servicio al público.

En el centro donde se realiza la investigación aquí presentada se utiliza un reactor anaerobio con deflectores. Se obtiene un agua tratada con concentraciones de salida que cumple con la normativa en vigor (NOM-002-SEMARNAP-1997) para ser descargada en el drenaje, pero no con las normas internacionales establecidas para su uso en riego (Cuadro I).

Existen reportes sobre el uso de reactores con deflectores para el tratamiento de aguas residuales domésticas (Hahn y Figueroa 2015, Reynaud y Buckley 2016) por la remoción de materia orgánica y sólidos suspendidos que pueden alcanzar; sin embargo, debido a que los efluentes de los reactores anaerobios contienen altas concentraciones de organismos coliformes, los humedales artificiales pueden ser una buena alternativa para mejorarlos (Galindo et al. 2019).

Con base en lo anterior, se planteó utilizar un humedal artificial de flujo subsuperficial con plantas de caña de azúcar y heliconia, como método de tratamiento terciario, debido principalmente a que los 
CUADRO I. DESEMPEÑO DEL REACTOR ANAEROBIO CON DEFLECTORES

\begin{tabular}{|c|c|c|c|c|c|}
\hline Parámetro & Promedio & Mínimo & Máximo & $\begin{array}{c}\text { NOM-003- } \\
\text { SEMARNAP-1997 }\end{array}$ & $\begin{array}{c}\text { Criterio de calidad de agua } \\
\text { para reúso en riego agrícola } \\
\text { (Unión Europea) }\end{array}$ \\
\hline $\mathrm{DBO}_{5}(\mathrm{mg} / \mathrm{L})$ & 87.5 & 40 & 120 & 20 & 25 \\
\hline DQO (mg/L) & 130.75 & 88 & 240 & NA & NA \\
\hline $\mathrm{SST}(\mathrm{mg} / \mathrm{L})$ & 75 & 40 & 125 & 20 & 35 \\
\hline $\begin{array}{l}\text { Coliformes fecales } \\
(\mathrm{NMP} / 100 \mathrm{~mL})\end{array}$ & 55000 & 10000 & 100000 & 240 & $\leq 100$ \\
\hline Grasas y aceites $(\mathrm{mg} / \mathrm{L})$ & 38.2 & 34.8 & 40.2 & 15 & NA \\
\hline
\end{tabular}

$\mathrm{DBO}_{5}$ : demanda bioquímica del oxígeno al quinto día, DQO: demanda química de oxígeno, SST: sólidos suspendidos totales, NMP: número más probable, NA: no aplica, NOM: Norma Oficial Mexicana.

Fuente: Cancino-Morales 2017.

humedales artificiales son sistemas cuya instalación y operación son de bajo costo (UN-HABITAT 2008).

El objetivo del presente trabajo fue establecer el desempeño de un sistema integrado por un reactor anaerobio con deflectores y un humedal artificial. En la evaluación se consideró la remoción de materia orgánica medida como demanda química de oxígeno (DQO) y demanda bioquímica de oxígeno al quinto día $\left(\mathrm{DBO}_{5}\right)$, sólidos suspendidos totales $(\mathrm{SST})$ y organismos coliformes totales y fecales.

\section{MATERIALES Y MÉTODOS}

\section{Descripción del sistema integrado de tratamiento}

El sistema estuvo formado por los componentes que se describen a continuación.

\section{Reactor anaerobio con deflectores}

Se utilizó un reactor anaerobio con deflectores que sirve como planta de tratamiento de aguas residuales (PTAR) en un centro de investigación. El reactor trata de 20 a $30 \mathrm{~m}^{3}$ /día y opera a un tiempo de retención hidráulica (TRH) de aproximadamente $24 \mathrm{~h}$. El reactor con deflectores consta de cuatro cámaras y tiene un volumen de $20 \mathrm{~m}^{3}$ (Fig. 1) (Chayes-Agustín 2013).

\section{Humedal artificial a escala piloto}

En investigaciones previas (Cancino-Morales 2017) se analizó el desempeño de humedales artificiales trasplantados con caña de azúcar (Saccharum officinarum L.) para el tratamiento del agua residual del mismo efluente utilizado en esta investigación en cuanto a la remoción de $\mathrm{DQO}, \mathrm{DBO}_{5}$ y $\mathrm{SST}$, usando dos TRH de 12 y $24 \mathrm{~h}$. El humedal tuvo el mejor con TRH de $24 \mathrm{~h}$, por lo cual se utilizó este último.

Se realizó la construcción del humedal tomando en cuenta los modelos utilizados en la literatura consultada (Konnerup et al. 2009, Ramachandra et al. 2017, Galindo et al. 2019). Se optó por un humedal artificial subsuperficial de flujo horizontal en un canal excavado en suelo con pendiente de $1 \%$. El canal tiene forma de trapecio invertido con las siguientes medidas: ancho menor en el fondo $(100 \mathrm{~cm})$ que en la parte superior $(126 \mathrm{~cm})$; largo inferior de 650 $\mathrm{cm}$ y superior de $670 \mathrm{~cm}$; altura menor $(40 \mathrm{~cm})$ a la entrada del humedal y mayor a la salida $(46.5 \mathrm{~cm})$. El esquema del humedal artificial se muestra en la

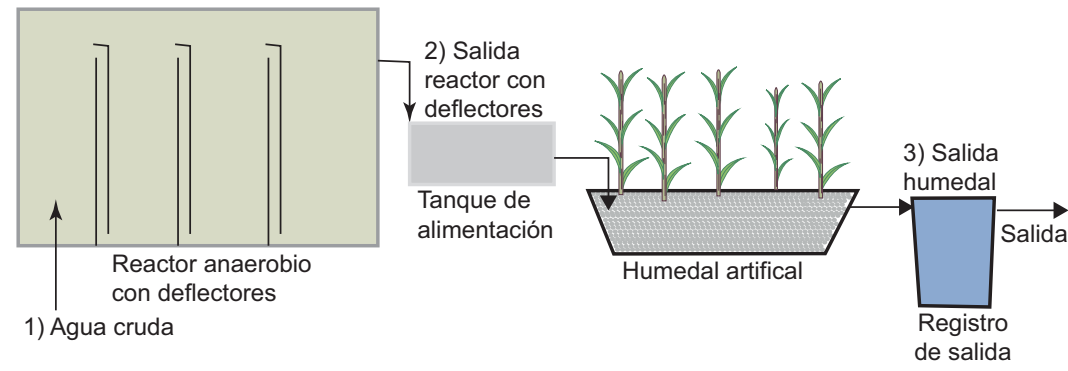

Fig. 1. Esquema del sistema acoplado reactor anaerobio de flujo ascendente-humedal artificial. 1, 2 y 3 representan los puntos donde se tomaron las muestras. 
Figura 1. El TRH del humedal fue de 24 h. Para impermeabilizar el canal se utilizó geomembrana de polietileno de alta densidad de $3 \mathrm{~mm}$ de espesor y para protegerla se utilizó una capa de arena de 10 $\mathrm{cm}$. Como material de soporte se utilizó granzón fino ( $5 \mathrm{~mm}$ de diámetro en promedio), triturado a partir de rocas extraídas de los ríos de la región. $\mathrm{Su}$ composición química no fue determinada.

Se trasplantaron un total de 18 plantas con una altura de $30 \mathrm{~cm}$ : nueve de caña de azúcar (S. officinarum L.) y nueve de heliconia (Heliconia psittacorum L.F.) de forma intercalada, dejando un espacio de $30 \mathrm{~cm}$ entre el borde libre del humedal y las plantas. Las plantas se colocaron en dos surcos con una separación de $65 \mathrm{~cm}$ entre cada surco y la misma distancia entre cada planta.

Se instalaron cuatro tubos de muestreo para dar seguimiento al humedal, los cuales consistieron en tramos de PVC (5.08 cm de diámetro) con perforaciones de $2 \mathrm{~mm}$ en las paredes de uno de sus extremos. Estos tubos se colocaron de manera vertical con el extremo perforado hacia abajo, de manera que el extremo con las perforaciones quedara en contacto con el material de soporte y el extremo sin perforaciones sobresaliera del soporte del humedal. El primero se instaló a $100 \mathrm{~cm}$ de la salida del humedal y el resto con una distancia de $100 \mathrm{~cm}$ entre cada uno. A fin de lograr una distribución uniforme del agua del humedal, la alimentación se llevó a cabo mediante un sistema de cuatro tubos PVC (5.08 cm de diámetro) colocados en paralelo a $20 \mathrm{~cm}$ del borde de la entrada y a una distancia de $23 \mathrm{~cm}$ entre ellos. A estos tubos se les realizaron perforaciones de $2 \mathrm{~mm}$ de diámetro en las paredes y se acoplaron a una sola fuente de alimentación, de manera que el agua fluyera por los cuatro tubos al mismo tiempo.

En el centro de investigación labora un total de 78 personas incluyendo personal académico, administrativo, de apoyo y mantenimiento, además de una población fluctuante de alrededor de 100 estudiantes de niveles medio, superior y posgrado. Cuenta con 25 laboratorios, cuatro colecciones (de entomología, hongos y un herbario), oficinas administrativas, 14 baños, una cafetería y cuatro departamentos que incluyen cocina y baño.

\section{Monitoreo de parámetros físicos y químicos}

Para determinar la eficiencia del sistema, se plantearon tres puntos de muestreo: la entrada del reactor anaerobio con deflectores, la salida del reactor con deflectores y la salida del humedal artificial. Se recolectó un litro de agua de cada uno de los puntos de muestreo y se analizaron los siguientes parámetros: $\mathrm{DBO}_{5}$ al quinto día por el método de dilución; $\mathrm{DQO}$;
SST por el método gravimétrico; organismos coliformes fecales por el método de fermentación en tubos múltiples, y pH mediante la técnica potenciométrica. Todos los análisis se realizaron de manera semanal utilizando métodos estandarizados (APHA 2017) por triplicado durante un periodo de 200 días.

El sistema acoplado reactor anaerobio con deflectores-humedal artificial se operó durante 200 días. Debido al crecimiento de las plantas de caña de azúcar fue necesario realizar un corte de éstas al día 165 de operación, lo cual se vio reflejado en el desempeño del humedal.

Las características de las aguas residuales crudas del centro de investigación fueron caracterizadas previamente de la siguiente manera: $\mathrm{DBO}_{5}=124.59$ $\pm 68.27 \mathrm{mg} / \mathrm{L} ; \mathrm{DQO}=259.09 \pm 143.80 \mathrm{mg} / \mathrm{L}$; $\mathrm{SST}=123.13 \pm 93.27 \mathrm{mg} / \mathrm{L})$. Se consideró realizar determinaciones del agua cruda hasta la estabilización del sistema a partir del día 115.

\section{RESULTADOS Y DISCUSIÓN}

\section{La remoción de $\mathrm{DBO}_{5}$ al quinto día fue insuficiente}

La dinámica de la $\mathrm{DBO}_{5}$ del sistema se muestra en la Figura 2. De manera general, la remoción promedio de este parámetro fue de $77.83 \pm 11.15 \%$ (Cuadro II) con un TRH de dos días (un día en el reactor anaerobio con deflectores y uno en humedal artificial). La remoción de $\mathrm{DBO}_{5}$ no fue suficiente para cumplir con los estándares nacionales e internacionales: $\leq 20 \mathrm{mg} / \mathrm{L}$ para la norma mexicana y $\leq 25 \mathrm{mg} / \mathrm{L}$ para la norma europea de agua clase B para riego de cultivos alimentarios que no entren en contacto con el agua, cultivo de alimentos que serán procesados o cultivo de alimento para ganado

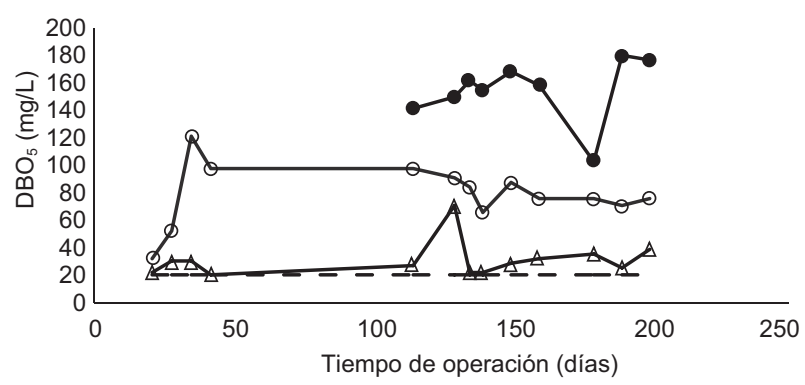

Fig. 2. Dinámica de la $\mathrm{DBO}_{5}$ del sistema acoplado reactor anaerobio con deflectores-humedal artificial. • agua sin tratamiento; $\circ$ efluente del reactor anaerobio con deflectores; $\Delta$ efluente del humedal artificial; -- Norma Oficial Mexicana para reúso de agua en riego de áreas verdes. 
CUADRO II. DESEMPEÑO DEL SISTEMAACOPLADO REACTOR ANAEROBIO CON DEFLECTORESHUMEDAL ARTIFICIAL EN LA REMOCIÓN DE CONTAMINANTES

\begin{tabular}{lccc}
\hline Parámetro \% de remoción & Promedio* & Mínimo & Máximo \\
\hline $\mathrm{DBO}_{5}$ del reactor anaerobio con deflectores & $48.73 \pm 12.10$ & 31.25 & 61.11 \\
$\mathrm{DBO}_{5}$ del humedal artificial & $58.71 \pm 15.71$ & 23.41 & 72.73 \\
$\mathrm{DBO}_{5}$ del sistema & $77.73 \pm 11.15$ & 53.33 & 85.80 \\
DQO del reactor anaerobio con deflectores & $40.50 \pm 10.53$ & 30.00 & 54.54 \\
DQO del humedal artificial & $61.42 \pm 20.80$ & 13.13 & 71.42 \\
DQO del sistema & $66.67 \pm 9.66$ & 50.55 & 83.33 \\
SST del reactor anaerobio con deflectores & $50.25 \pm 6.75$ & 42.70 & 57.89 \\
SST del humedal artificial & $75.70 \pm 7.72$ & 62.32 & 83.63 \\
SST del sistema & $88.38 \pm 2.25$ & 84.11 & 90.08 \\
Coliformes totales del reactor anaerobio con deflectores & $55.31 \pm 12.00$ & 50.41 & 79.83 \\
Coliformes totales del humedal artificial & $99.53 \pm 0.76$ & 97.98 & 99.79 \\
Coliformes totales del sistema & $99.78 \pm 0.38$ & 99.00 & 99.96 \\
Coliformes fecales del reactor anaerobio con deflectores & $99.98 \pm 4.98$ & 87.39 & 99.00 \\
Coliformes fecales del humedal artificial & $95.17 \pm 4.98$ & 90.00 & 99.62 \\
Coliformes fecales del sistema & $99.77 \pm 0.39$ & 99.00 & 99.99 \\
\hline
\end{tabular}

Nota: los valores mostrados corresponden a 13 muestras con tres repeticiones.

*Promedio \pm desviación estándar.

$\mathrm{DBO}_{5}$ : demanda bioquímica del oxígeno al quinto día, DQO: demanda química de oxígeno, SST: sólidos suspendidos totales.

(Alcande-Sanz y Gawlik 2017). Sin embargo, la concentración se mantuvo por debajo de $40 \mathrm{mg} / \mathrm{L}$.

Los mecanismos de remoción de $\mathrm{DBO}_{5}$ dentro del reactor con deflectores son principalmente la retención de la materia en el lodo, la degradación microbiológica y la sedimentación (Hahn y Figueroa 2015). No obstante, la remoción obtenida por el reactor con deflectores fue en promedio menor al 50 \% (Cuadro II). Este resultado evidencia algún problema operacional, por lo que se requiere, entre otras acciones, revisar la cantidad de lodo presente en las cámaras del reactor. Por su parte, la remoción de este parámetro en el humedal fue moderada $(<60 \%)$, posiblemente debido a exudados de las plantas, ya que al parecer hubo algún grado de competencia entre las plantas de heliconia y de caña.

Ávila et al. (2015) reportaron concentraciones menores de $\mathrm{DBO}_{5}$ a la salida de un sistema acoplado de un tanque Imhoff, tres tipos de humedales artificiales y una laguna de estabilización. En un TRH de 7.4 días y con una entrada de $\mathrm{DBO}_{5}$ de $320 \mathrm{mg} / \mathrm{L}$, obtuvieron una remoción de $99 \%$ (la $\mathrm{DBO}_{5}$ del efluente fue de $4 \mathrm{mg} / \mathrm{L}$ ). No obstante, es posible obtener mejores resultados debido al mayor número de unidades y mayor TRH utilizado.

Nuestros resultados contrastan con los de Tufaner (2020), quienes utilizaron un sistema acoplado reactor anaerobio de flujo ascendente (RAFA)-humedal artificial a escala de laboratorio. El RAFA de 6 L operó con una DQO inicial de $1000 \mathrm{mg} / \mathrm{L}, \mathrm{DBO}_{5}$ de $167 \mathrm{mg} / \mathrm{L}$ y un humedal artificial híbrido de $2500 \mathrm{~L}$ con dos secciones, una de flujo superficial y la segunda de flujo vertical. Por medio de este sistema se obtuvo una remoción de $\mathrm{DBO}_{5}$ del sistema completo de $83 \%$ en un TRH de $\approx 96$ días, mayor que en el presente trabajo (si bien el TRH también fue mayor que el empleado en este trabajo).

\section{La remoción de DQO en ambas partes del sistema fue moderada}

La DQO del influente fluctuó entre 270 y $380 \mathrm{mg} / \mathrm{L}$ (Fig. 3). El sistema presentó un promedio de remoción de $66.67 \pm 9.66 \%$. La normativa europea

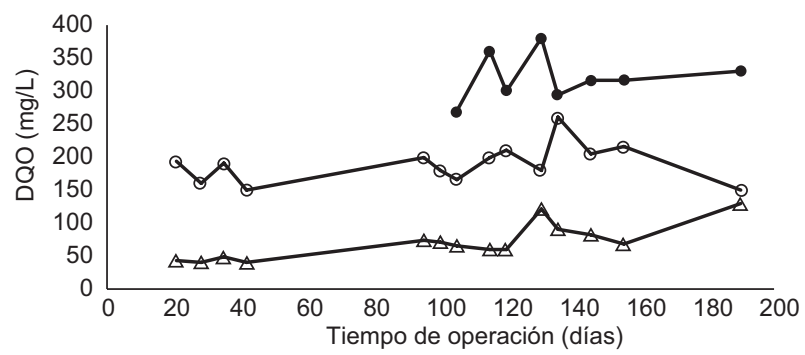

Fig. 3. Dinámica de la demanda química de oxígeno al quinto día (DQO) del sistema acoplado reactor anaerobio con deflectores-humedal artificial. $\bullet$ agua sin tratamiento; 0 efluente del reactor anaerobio con deflectores; $\Delta$ efluente del humedal artificial. 
contempla la remoción de al menos $70 \%$ con relación a este parámetro y una concentración de salida menor o igual a $125 \mathrm{mg} / \mathrm{L}$ para ser descargado a aguas receptoras.

La concentración de la DQO a la salida del humedal se estabilizó alrededor de $60 \mathrm{mg} / \mathrm{L}$. Como puede observarse, después del día 165 se obtuvo el máximo valor debido al efecto del corte de las plantas de caña en el humedal, es decir, un efecto negativo en la remoción. Se obtuvieron en todos los casos remociones menores a $80 \%$. Lu et al. (2016) reportaron concentraciones de salida de DQO similares (de 51.2 a $93.4 \mathrm{mg} / \mathrm{L}$ ) utilizando humedales artificiales con diferentes materiales de empaque a un TRH de 7 días, lo cual constituye un indicio adicional de que la DQO del efluente sería menor si se incrementara el TRH del presente trabajo.

\section{El uso del humedal artificial permite una alta remoción de SST}

El promedio de la concentración de SST a la salida del sistema fue de $11.44 \pm 3.38 \mathrm{mg} / \mathrm{L}$. Las concentraciones alcanzadas cumplieron con la normativa europea para agua clase B $(35 \mathrm{mg} / \mathrm{L})$ así como la norma mexicana para riego de áreas verdes $(20 \mathrm{mg} / \mathrm{L})$. La remoción de este parámetro fluctuó entre 84 y $90 \%$ (Fig. 4).

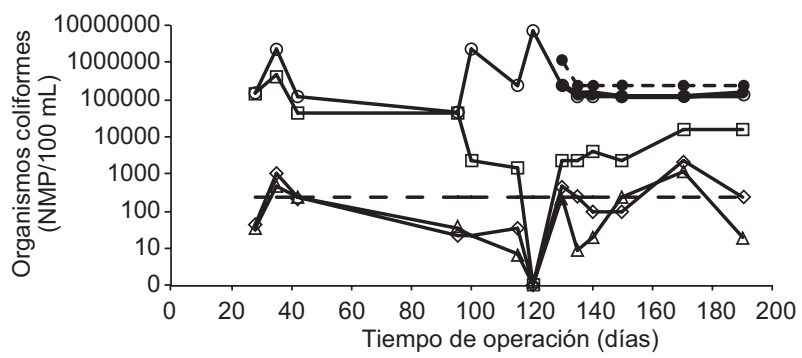

Fig. 5. Dinámica de organismos coliformes del sistema acoplado digestor anaerobio-humedal artificial. $\bullet$ organismos coliformes totales (OCT) del agua sin tratamiento; $\bullet$ organismos coliformes fecales (OCF) del agua sin tratamiento; - OCT de efluente del reactor anaerobio con deflectores; $\square$ OCF del reactor anaerobio con deflectores; $\diamond$ OCT del efluente del humedal artificial; $\triangle$ OCF del efluente del humedal artificial; -- Norma Oficial Mexicana para reúso de agua en riego de áreas verdes.

Dentro del reactor con deflectores, la remoción de sólidos se lleva a cabo mediante dos procesos: la degradación de materia orgánica por microorganismos y la sedimentación de sólidos (Hahn y Figueroa 2015). Por su parte, entre los mecanismos que permiten la alta remoción de sólidos generada por el humedal artificial se pueden mencionar la degradación biológica de los sólidos, la sedimentación, la adsorción en la materia orgánica, la retención en las raíces y la adsorción al soporte (Galindo et al. 2019).

Es importante señalar que al parecer la remoción de este parámetro no se vio alterada por el corte de las plantas, probablemente debido a que no se afectaron físicamente las raíces, que en este caso parecen funcionar como una barrera física para la retención de SST.

Algunos autores reportan concentraciones de salida de alrededor de $4 \mathrm{mg}$ de SST/L con TRH de 5 días (Ávila et al. 2015). Esto sugiere que en aguas residuales de baja carga, los SST son materiales de fácil eliminación y que el TRH utilizado en el presente trabajo es suficiente para removerlos.

\section{Los organismos coliformes totales y fecales son removidos principalmente en el humedal artificial}

La remoción de organismos coliformes totales y fecales se realizó principalmente en el humedal artificial, siendo influenciada por mecanismos como sedimentación, adsorción en materia orgánica retenida en la matriz del humedal, destrucción de bacterias por fotooxidación y exposición de los organismos coliformes a la acción biocida de compuestos exudados por las plantas (Weber y Legge 2008, Wu et al. 2016)

De acuerdo con los lineamientos estipulados por la organización mundial de la salud (WHO 2006), considerando una concentración de bacterias patógenas de $1 \times 10^{6} / 100 \mathrm{~mL}$, el tratamiento de agua residual debe ser capaz de remover 3 unidades logarítmicas (equivalentes a una concentración de 1 $\times 10^{3} / 100 \mathrm{~mL}$ ) para agua no clorada. En el presente trabajo se logró alcanzar este requerimiento después de 100 días de operación (Fig. 5).

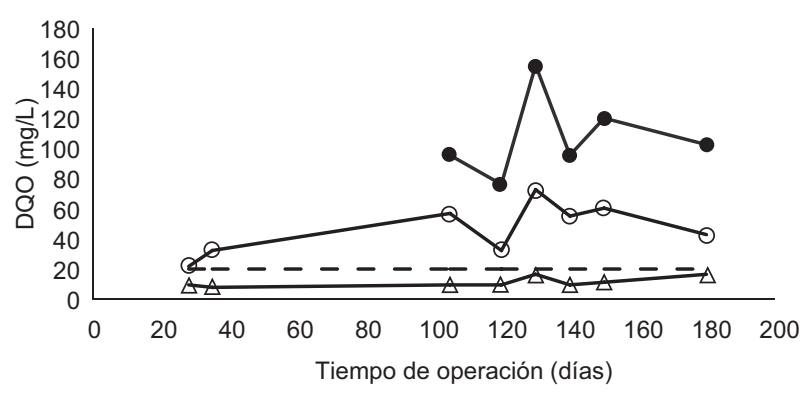

Fig. 4. Dinámica de sólidos suspendidos totales (SST) del sistema acoplado reactor anaerobio-humedal artificial. • agua sin tratamiento; $\circ$ efluente del reactor anaerobio con deflectores; $\Delta$ efluente del humedal artificial; -- Norma Oficial Mexicana para reúso de agua en riego de áreas verdes. 
Respecto de la normativa europea, la concentración de $E$. coli debe ser $\leq 10 \mathrm{UFC} / 100 \mathrm{~mL}$ para agua clase A (que puede usarse para riego de productos alimentarios que entran en contacto directo con el agua) $\mathrm{o} \leq 100 \mathrm{UFC} / 100 \mathrm{~mL}$ para agua clase $\mathrm{B}$. Por su parte, la norma mexicana señala que el agua residual tratada para reúso en riego de áreas verdes no debe superar los 240 NMP coliformes fecales/100 mL. En los primeros días, la concentración de organismos coliformes totales a la salida del humedal fue superior a $1 \times 10^{3} \mathrm{NMP} / 100 \mathrm{~mL}$. Sin embargo, después de 50 días de operación, la cantidad de organismos coliformes fecales se estabilizó y cumplió con lo dispuesto en la norma mexicana (Fig. 5).

El corte de las plantas de caña afectó la remoción de organismos coliformes, pero a los 190 días de operación su concentración disminuyó nuevamente y se cumplió con lo establecido en la norma mexicana.

Es posible que la remoción de coliformes se haya visto afectada negativamente por un cambio en la fisiología de las plantas ocasionada por la poda, al alterar las interacciones y comunidades microbianas en el humedal (Alusafi et al. 2017), ya que no se afectó físicamente el soporte.

Wu et al. (2016) reportaron la remoción de organismos coliformes fecales de $1 \times 10^{5}$ a $10^{8}$ UFC/100 $\mathrm{mL}$, y obtuvieron efluentes con concentraciones desde $1 \times 10^{3} \mathrm{UFC} / 100 \mathrm{~mL}$ hasta $1 \times 10^{5} \mathrm{UFC} / 100 \mathrm{~mL}$ con TRH de 1.5 a 5 días. Ávila et al. (2015) reportaron concentraciones de organismos coliformes similares a las encontradas en el presente trabajo: con TRH de 5 días, obtuvieron efluentes con concentraciones $<$ $40 \mathrm{UFC} / 100 \mathrm{~mL}$. La alta eficiencia de remoción de organismos coliformes se logró con un TRH relativamente corto (2 días para todo el sistema reactor anaerobio con deflectores-humedal artificial) debido probablemente al tipo de plantas utilizadas.

Entre los mecanismos de remoción de organismos patógenos en los humedales artificiales se menciona la muerte de organismos por condiciones desfavorables del medio, exposición a compuestos nocivos para los patógenos, interacción con el medio de soporte y exposición a luz ultravioleta (Weber y Legge 2008, Alufasi et al. 2017).

Tunçsiper et al. (2012) reportaron la remoción de coliformes totales y fecales en un rango de 88 a $98 \%$ con concentraciones iniciales de $1.98 \times 10^{4}$ coliformes fecales y $1.44 \times 10^{5} \mathrm{UFC} / 100 \mathrm{~mL}$ coliformes totales. Estos autores utilizaron TRH de 1.32, $1.65,2.20$ y 4.40 días y concluyeron que la remoción de patógenos no mejora después de un TRH de 2 días. Cabe señalar que estos autores trabajaron con concentraciones iniciales de organismos coliformes similares a las del presente trabajo y el porcentaje de remoción que se alcanzó es ligeramente más bajo. Para concentraciones iniciales más altas de organismos coliformes, se puede mencionar el trabajo de Zurita y Carreón-Álvarez (2015) quienes alcanzaron remociones de 96.92 y $99.99 \%$ para coliformes totales y fecales, respectivamente, cuando las concentraciones iniciales fueron de 2.5 y $1.6 \times 10^{6} \mathrm{NMP} / 100 \mathrm{~mL}$ para coliformes totales y fecales, respectivamente. Estos autores trabajaron con sistemas de tratamiento consistentes en un humedal artificial unido a una laguna de estabilización, un humedal artificial horizontal unido a un humrdal de flujo vertical, y un humedal de flujo vertical unido a un humedal de flujo horizontal. El TRH que utilizaron fue de 3 días.

\section{El pH del sistema se mantuvo neutro}

El agua cruda tenía un $\mathrm{pH}$ de 7.64 (ligeramente básico), el cual se neutralizó a la salida del reactor anaerobio con deflectores (7.13). La alcalinidad total promedio a la salida del reactor anaerobio con deflectores fue de $380 \mathrm{mg} \mathrm{CaCO}_{3} / \mathrm{L}$ y un índice de amortiguamiento (alcalinidad intermedia/alcalinidad total) de 0.29 , que es una alcalinidad suficiente para mantener el $\mathrm{pH}$ neutro y un indicador de la estabilidad del proceso.

A la salida del humedal el agua tuvo $\mathrm{pH}$ cercano a la neutralidad (6.95), lo cual cumple con la norma mexicana (NOM-003-SEMARNAP-1997). Este parámetro no se considera en la norma europea.

\section{CONCLUSIONES}

Con el sistema acoplado reactor anaerobio con deflectores-humedal artificial fue posible remover SST y organismos coliformes fecales, generando efluentes que cumplen con lo establecido para estos parámetros por las normas europea y mexicana para riego en áreas verdes.

Con el TRH utilizado no fue posible cumplir con las regulaciones europea y mexicana para la $\mathrm{DBO}_{5}$.

El sistema utilizado tiene potencial para el tratamiento de aguas residuales en centros de investigación e instituciones similares si se mejora el control del crecimiento de plantas y el TRH.

\section{AGRADECIMIENTOS}

Los autores agradecen el financiamiento obtenido por el Fondo de Investigación Científica y Desarro1lo Tecnológico de El Colegio de la Frontera Sur 
(FID-784), acuerdo 08/1E/18-S. Asimismo, agradecen al Dr. Alessandro Carmona Martínez por su apoyo en la revisión del presente documento.

\section{REFERENCIAS}

Alcande-Sanz L. y Gawlik B.M. (2017). Minimum quality requirements for water reuse in agricultural irrigation and aquifer recharge. Towards a legal instrument on water reuse at EU level. Oficina de Publicaciones de la Unión Europea, 57 pp. https://doi.org/10.2760/804116

Alufasi R., Gere J., Chakauya E., Lebea P, Parawira W. y Chingwaru W. (2017). Mechanisms of pathogen removal by macrophytes in constructed wetlands. Environ. Technol. Rev. 6 (1), 135-144. https://doi.org $/ 10.1080 / 21622515.2017 .1325940$

APHA (2017). Standard methods for the examination of water and wastewater. 23rd ed. American Public Health Association, Washington DC, $1325 \mathrm{pp}$.

Ávila C., Bayona J.M., Martín I., Salas J.J. y García J. (2015). Emerging organic contaminant removal in a full-scale hybrid constructed wetland system for wastewater treatment and reuse. Ecol. Eng. 80, 180116. https://doi.org/10.1016/j.ecoleng.2014.07.056

Cancino-Morales V.A. (2017). Tratamiento de aguas residuales por un sistema piloto de humedales artificiales para la reutilización en riego de áreas verdes. Tesis de licenciatura. Instituto Tecnológico de Tapachula, Tapachula, Chiapas, México, 45 pp.

Chayes-Agustín M.A. (2013). Desempeño de un reactor UASB compartimentado al tratar agua residual de baja carga. Tesis de maestría. El Colegio de la Frontera Sur, Tapachula, Chiapas, México, 67 pp.

Dong-Qing Z., Jinadasa K.B.S.N., Gersberg R.M., Liu Y., Tan S.K. y Ng W.J. (2015). Application of constructed wetlands for wastewater treatment in tropical and subtropical regions. J. Environ Sci. 30, 30-46. https:// doi.org/10.1016/j.jes.2014.10.013

Galindo Montero A., Pimienta Serrano E.V. y Pérez Montiel J.I. (2019). Sanitary landfill leachate treatment with double chamber anaerobic reactor in series with constructed wetland. Environ. Process. 6, 695-712. https://doi.org/10.1007/s40710-019-00380-z

Hahn M. y Figueroa L.A. (2015). Pilot scale application of anaerobic baffled reactor for biologically enhanced primary treatment of raw municipal wastewater. Water Res. 87 (15), 494-502 https://doi.org/10.1016/j. watres.2015.09.027

Hamoda M.F., al-Gusain I. y al-Mutairi N.Z. (2004). Sand filtration of wastewater for tertiary treatment and water reuse. Desalination 164, 203-201. https:// doi.org/10.1016/j.desal.2008.12.049
WWAP 2017. Facts and figures. En: The United Nations World Water Development Report 2017: Wastewater, the untapped resource. United Nations World Water Assessment Programme. UNESCO, París, 12 pp.

Konnerup D., Koottatep T. y Brix H. (2009). Treatment of domestic wastewater in tropical, subsurface flow constructed wetlands planted with Canna and Heliconia. Ecol. Eng. 35, 248-157. https://doi.org/10.1016/j. ecoleng.2008.04.018

Lu S., Zhang X., Wang J. y Pei L. (2016). Impacts of different media on constructed wetlands for rural household sewage treatment. J. Clean. Prod. 127, 325-330. https:// doi.org/10.1016/j.jclepro.2016.03.166

Mayram B. y Büyükgüngör H. (2019). Wastewater reclamation and reuse in Turkey: Opportunities and challenges. J. Water Process. Eng. 30. https://doi. org/10.1016/j.jwpe.2017.10.001

Ramachandra T.V., Bhat S. y Shivamurthy V. (2017). Constructed wetlands for tertiary treatment of wastewater. ENVIS Technical Report 124. Energy and Wetlands Research Group, CES, Indian Institute of Science, Bangalore, India, $70 \mathrm{pp}$.

Reynaud N. y Buckley C.A. (2016). The anaerobic baffled reactor (ABR) treating communal wastewater under mesophilic conditions: A review. Water Sci. Technol. 73 (3), 463-478. https://doi.org/10.2166/ wst.2015.539

Salgot M. y Folch M. (2018). Wastewater treatment and wastewater reuse. Curr. Op. Environ. Sci. Health 2, 64-74. https://doi.org/10.1016/j.coesh.2018.03.005

SEMARNAP (1998). Norma Oficial Mexicana NOM003-ECOL-1997. Que establece los límites máximos permisibles de contaminantes para las aguas residuales tratadas que se reúsen en servicios al público. Secretaría del Medio Ambiente, Recursos Naturales y Pesca. Diario Oficial de la Federación, 21 de septiembre.

Tunçsiper B., Ayaz S. y Akcca L. (2012). Coliform bacteria removal from septic wastewater in a pilotscale combined constructed wetland system. Environ. Eng. Manag. J. 11 (10), 1873-1879. https://doi. org/10.30638/eemj.2012.233

Tufaner F. (2020). Post-treatment of effluents from UASB reactor treating industrial wastewater sediment by constructed wetland. Environ. Technol. 41 (7), 912920. https://doi.org/10.1080/09593330.2018.1514073

UN-HABITAT (2008). Constructed wetlands manual. Water for Asian Cities Programme, UN-HABITAT, Nepal, Katmandú, 102 pp.

Weber K.P. y Legger R.L. (2008). Pathogen removal in constructed wetlands. En: Wetlands: Ecology, conservation \& restoration. (Russo R.E., Ed.). Nova Science Publisher, 177-211. 
WHO (2006). Guidelines for the safe use of wastewater, excreta and greywater. Vol 1. World Health Organization, Francia, $124 \mathrm{pp}$.

Wu S., Carvalho P.N., Müller J.A., Manoj R.V. y Dong R. (2016) Sanitation in constructed wetlands: A review on the removal of human pathogens and fecal indicators. Sci. Total. Environ. 541, 8-22. https://doi. org/10.1016/j.scitotenv.2015.09.047
Zurita F. y Carreón-Álvarez A. (2015) Performance of three pilot-scale hybrid constructed wetlands for total coliforms and Escherichia coli removal from primary effluent - A 2-year study in a subtropical climate. J. Water Health. 13 (2), 446-458. https://doi.org/10.2166/ wh.2014.135 